\title{
Characterisation of protease resistance mutations in a South African paediatric cohort with virological failure, 2011 - 2017
}

\author{
Z Makatini, ${ }^{1,2}$ MSc, MB ChB, DTM\&H, Dip HIV Man, FCPath (SA) Viro; S Mda, ${ }^{1}$ MB ChB, PhD; O Towobola, ${ }^{1} \mathrm{PhD} ; \mathbf{H}$ Mthiyane, ${ }^{2} \mathrm{MSc}$; \\ P Miles, ${ }^{3} \mathrm{MSc}$; J T Blackard, ${ }^{4} \mathrm{PhD}$ \\ ${ }^{1}$ Department of Virology, Faculty of Medicine, Sefako Makgatho Health Sciences University, Pretoria, South Africa \\ ${ }^{2}$ Department of Virology, Faculty of Health Sciences, University of the Witwatersrand, Johannesburg, South Africa \\ ${ }^{3}$ Waltham Forest Clinical Commissioning Group, Leytonstone, London, UK \\ ${ }^{4}$ Department of Internal Medicine, College of Medicine, University of Cincinnati, Ohio, USA
}

Corresponding author: Z Makatini (zinhle@wol.co.za)

\begin{abstract}
Background. Advances in HIV management have improved treatment outcomes in the HIV-infected population. However, these advances have not been without multifaceted challenges. In sub-Saharan Africa, their impact is reflected in the increased emergence of HIV drug resistance mutations. With the rise in exposure of children to protease inhibitors (PIs), the possibility of increasing PI resistance remains a concern.

Objectives. To describe a group of antiretroviral-experienced children with PI drug resistance mutations after failure on first- or second-line regimens in a public sector setting in South Africa.

Methods. This was a retrospective cohort study of 22 children perinatally infected with HIV who had HIV genotyping conducted between January 2011 and December 2017.

Results. Of the 236 children who had HIV genotyping conducted, 22 (9.3\%) had evidence of HIV PI resistance mutations. Twenty-one of the 22 children (95.5\%) had major mutations in the protease region of the HIV genome. Of these children, 66.7\% (14/21) had loss of response to both boosted lopinavir and atazanavir, with boosted darunavir remaining susceptible in only $12(57.1 \%)$. The most frequent major PI mutations were V82A (76.2\%), M46I/M46L (76.2\%), I54V (62.0\%) and L76V (33.3\%).

Conclusions. We observed a high rate of PI resistance mutations, with a resulting loss of PIs that could be used in construction of thirdline regimens. To build on improvements from the introduction of antiretroviral therapy, increased efforts are needed by both health professionals and caregivers to improve adherence measures in children perinatally infected with HIV.
\end{abstract}

S Afr Med J 2019;109(7):511-515. DOI:10.7196/SAMJ.2019.v109i7.13705

The introduction and scale-up of combination antiretroviral therapy (cART) has resulted in a significant decrease in morbidity and mortality rates in children perinatally infected with HIV ${ }^{[1,2]}$ These children are now surviving into adolescence and adulthood with lifelong chronic HIV infection. ${ }^{[3]}$ Challenges persist, however, in part owing to the limited availability of antiretroviral treatment options for paediatric patients. ${ }^{[4]}$ Further, inequalities persist in the South African (SA) public sector healthcare system, and limited clinical HIV expertise remains a key concern. ${ }^{[5]}$ In poorly resourced public sector settings in SA, with higher rates of virological failure ${ }^{[6-8]}$ and longer duration of a failing regimen, ${ }^{[4,9,10]}$ the emergence of drug resistance mutations poses a threat to future antiretroviral options.

SA national antiretroviral guidelines for the treatment of HIVinfected children have evolved from cART initiation with stavudine $(\mathrm{d} 4 \mathrm{~T})$ as part of the nucleoside reverse transcriptase inhibitor (NRTI) backbone to abacavir (ABC). ${ }^{[1]}$ Single dose-ritonavir (RTV) was routinely prescribed to children aged $<3$ years for the first 3 years of the antiretroviral roll-out programme in SA. ${ }^{[12]}$ Prior exposure to RTV-based regimens has been reported to be associated with suboptimal treatment responses, thus increasing the risk of acquisition of protease inhibitor (PI) resistance mutations. ${ }^{[13]}$ Boosted lopinavir (LPV) for children aged $<3$ years and efavirenz (EFV) for children aged $>3$ years has remained unchanged. A number of strategies, including lamivudine (3TC) monotherapy, have been widely employed to manage the complex adherence challenges in children failing first- or second-line therapy in the public sector setting. As a result, a large proportion of children are exposed to a wide array of antiretroviral agents with a potential of emergence of HIV drug resistance, resulting in limited options for the future. Access to salvage therapy in the SA public sector was implemented in 2015 by the National Department of Health and allows for construction of an appropriate regimen determined by an HIV genotype test. ${ }^{[11]}$

PIs are an important foundation for the management of HIVinfected children in SA. The type of PI exposure and whether unboosted PIs are used influence HIV resistance PI mutations and patterns that emerge. ${ }^{[14]}$ Despite the high genetic barrier of PIs, major PI resistance mutations have been reported in several SA studies (involving 89, 75 and 50 children), with frequencies of $6.3 \%, 10.7 \%$ and $14 \%$, respectively. ${ }^{[14-16]}$

\section{Objectives}

The current study focuses on a group of heavily experienced children managed in the public sector with resource constraints, with virological failure of either first-line or second-line regimens, longer duration of a failing regimen, exposure to various treatment strategies to mitigate virological failure, and the risk of increasing acquisition of PI resistance.

\section{Methods \\ Study population}

This was a retrospective cohort study of children perinatally infected with HIV attending Dr George Mukhari Academic Hospital 
(DGMAH), a public sector hospital located outside Pretoria, SA. Approximately 2200 children are actively managed in the DGMAH HIV paediatric clinic. Children are transferred to the adult HIV clinic on reaching the age of 16 years. The HIV package of care includes routine monthly clinic visits, follow-up CD4+ T-cell counts and HIV viral load (VL) testing, as well as safety bloods as defined by the SA National Antiretroviral Treatment Guidelines. ${ }^{[12]}$ Ongoing academic research activities provided access to HIV genotyping for individuals with a VL $>1000$ copies $/ \mathrm{mL}$. The majority of the paediatric attendees were initiated on an ABC with 3TC NRTI backbone with either EFV or boosted LPV depending on age. However, a number of children were initiated on treatment prior to 2010 and remained on $\mathrm{d} 4 \mathrm{~T}$ with 3TC as part of the NRTI backbone. Enrolment of the cohort was through convenience sampling of children aged $<16$ years who had been on cART for $\geq 1$ year, had developed virological failure, and had HIV genotyping conducted from January 2011 to December 2017. Children with missing clinical files or HIV genotype reports conducted prior to 2011 were excluded from the cohort.

\section{Ethical considerations}

The Sefako Makgatho Health Sciences University Research and Ethics Committee approved collection of clinical and genotype data (ref. no. MREC/P/83/2009). Written informed consent was obtained from each child's caregiver.

\section{HIV genotyping}

HIV genotyping was performed using an in-house drug resistance assay. Briefly, a $1.7 \mathrm{~kb}$ amplicon was generated by reverse transcriptase (RT)-initiated polymerase chain reaction of the entire protease (PR) and partial RT-coding regions. The amplicon was sequenced using five primers from codons 1 - 99 of PR and codons 1 - 230 of RT. Sequencing was performed with either an ABI PRISM 3730 or an ABI PRISM 3100-Avant Genetic Analyzer (Applied Biosystems, USA). To identify drug resistance mutations and predict drug susceptibility, the Stanford HIV Drug Resistance Database version 8.3 (Stanford University, USA) was used. Susceptibility to boosted LPV, atazanavir (ATV) and darunavir (DRV) was determined using the penalty score where, from the total score for each of the three PIs, five levels of inferred drug resistance were reported: susceptible, potential lowlevel resistance, low-level resistance, intermediate resistance and high-level resistance.

\section{Statistical analysis}

Clinical baseline and demographic variables were compared using medians with interquartile ranges (IQRs) for numerical data or proportions for categorical data, with genotype data presented as proportions. A two-sided Fisher's exact test was used to compare the association of categorical variables, and $p$-values $<0.05$ were considered statistically significant. Statistical analysis was performed using GraphPad Prism 6 (GraphPad Software, USA).

\section{Results \\ Patient characteristics}

Demographic and baseline clinical characteristics are presented in Tables 1 and 2. Of 236 children who had HIV genotyping conducted over the 6-year study period, $22(9.3 \%)$ had evidence of PI HIV resistance mutations and were included in the study; $54.5 \%$ of all children were female. World Health Organization (WHO) clinical stage at cART initiation was III or IV in $91.0 \%$ of the children. WHO stage III was the most common stage at $77.2 \%$, representing most of the children noted to have been placed on concurrent TB treatment at cART initiation. The median age at initiation was 3 years (IQR 1.25 -
8.6), with most of the children (45.5\%) initiating cART between the ages of 0 and 3 years and $\sim 14 \%$ at an advanced age of $10-16$ years. All children were documented to have been infected perinatally. Prior to treatment initiation, $40.9 \%$ of children had laboratory evidence of severe immunosuppression, with CD4+ T-cell percentages between $0 \%$ and $10 \%$. Review of CD4+ T-cell absolute counts revealed that $27.3 \%$ had CD4+ T-cell counts $<100$ cells $/ \mu \mathrm{L}$. Overall, the median duration of a failing regimen was 22 months (IQR 6 - 66), and the median VL at genotype was $4.5 \log _{10}$ copies $/ \mathrm{mL}$.

\section{Virological failure}

The median age of all 22 children at genotyping was 8 years (IQR 4.35 - 11.7), with a duration of 22 months on a failing regimen. The median HIV VL at failure was $4.52 \log _{10}$ (IQR 3.38 - 4.8). All 22 children with PI resistance mutations had been on a boosted LPV regimen at the time of genotyping. The predominant NRTI backbone in use in this cohort was zidovudine (AZT) with 3TC $(n=7,31.8 \%)$ and $\mathrm{ABC}$ with $3 \mathrm{TC}(n=7,31.8 \%)$. The remaining children were on AZT with $\mathrm{ABC}(n=5,22.7 \%)$ and d4T with $3 \mathrm{TC}(n=3,13.6 \%)$. In addition, 6 of the children (22.3\%) had prior exposure to single-dose RTV for a median duration of 3 months as well as 3TC monotherapy as a holding regimen.

\section{Drug resistance mutations}

All genotyped samples belonged to HIV subtype C. Twenty-one of the 22 children (95.5\%) had major mutations in the PR region. The distribution of all PI mutations is reflected in Figs 1 and 2. The most frequently occurring major PI mutations were V82A (76.2\%), I54V (62.0\%), M46I (52.4\%), L76V (33.3\%) and M46L (23.8\%). The remaining major PI mutations $I 47 \mathrm{~A}, I 84 \mathrm{~V}$ and $V 32 I$ were detected at frequencies of $9.5 \%, 4.7 \%$ and $4.7 \%$, respectively. The median number of major PI mutations was 3 (IQR 2 - 4), with the predominance of 3 mutations seen in 6 children. The major LPV resistance mutations detected were M46I, L76V and V82A, occurring alone or in combinations of up to 5 mutations in 7 children. Fourteen of the 21 children with major mutations (66.7\%) experienced loss of activity to both boosted LPV and ATV. However, boosted DRV remained susceptible in 12 (57.1\%).

Mutations classified as 'accessory' PI resistance mutations by the IAS (International Antiviral Society)-USA were detected in 10/22 children (45.5\%), with only one (4.5\%) having a Q58E non-

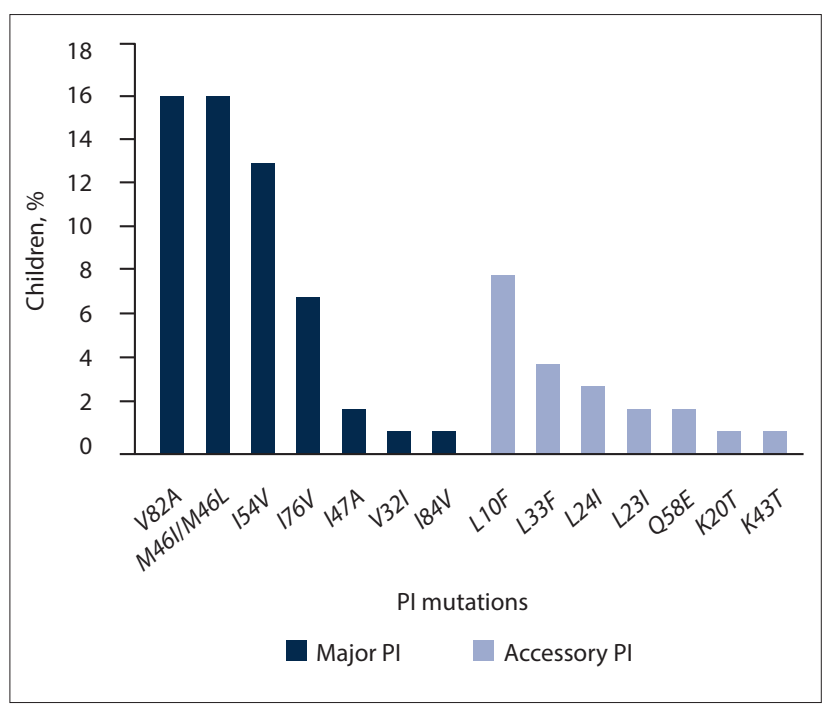

Fig. 1. Overall distribution of major and accessory PI mutations. $(P I=$ protease inhibitor). 
polymorphic accessory mutation exclusively. 'Other' PI mutations $A 71 \mathrm{~V}$ and $L 10 \mathrm{~V}$ were observed with a frequency of 2 each. Further

analysis of children with prior exposure to RTV revealed no association between duration of single-dose RTV and emergence

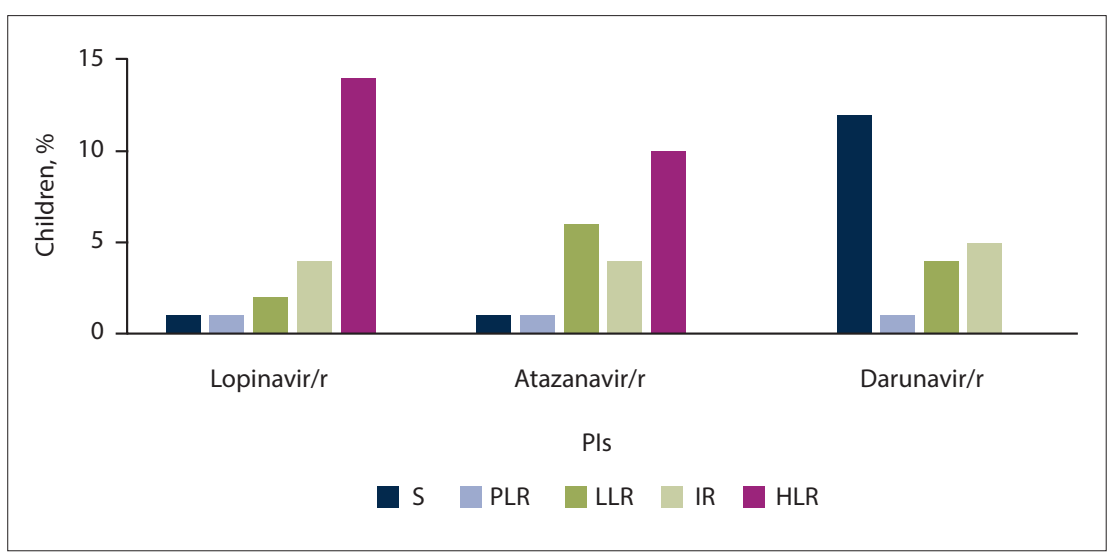

Fig. 2. Proportions of children with S PIs (penalty score $<10$ ) and PLR (penalty score $10-14)$, LLR (penalty score 15 - 29), IR (penalty score 30 - 59) and HLR (penalty score $>60)$. (PI = protease inhibitor; $S=$ susceptible; $P L R=$ potential low-level resistance; $L L R=$ low-level resistance; $I R=$ intermediate resistance; $H L R=$ high-level resistance; $r=$ ritonavir.) of PI mutations $(p=0.275)$. There was no association between duration of a failing regimen $(<2$ years v. $>2$ years on failing regimen) and the emergence of additional PI mutations ( $p=0.6032$ ).

The most common NRTI mutation was the $M 184 \mathrm{~V}$ mutation, detected at a frequency of 17 (77.3\%). L74V and $L 74 I-$ both with compensatory effects on viral fitness - were detected in children on $\mathrm{ABC}$, with a frequency of $2(9.1 \%)$ and $1(4.5 \%)$, respectively. The combination of $L 74 \mathrm{~V}$ plus $M 184 V$ occurred in 3 children (13.6\%) in our cohort. Viruses carrying the $K 65 R$ resistance mutation were observed in only 3 children (13.6\%). Of interest is that none of these children were documented to have been on tenofovir disoproxil fumarate (TDF), but all had exposure to $\mathrm{ABC}$ at the time of HIV genotyping. Thymidine analogue mutations (TAMs) were observed in $63.6 \%$ of the children, with 4 (18.2\%), 3 (13.6\%)

\begin{tabular}{|c|c|}
\hline Characteristic & All children $(N=22)$ \\
\hline Gender female, $n(\%)$ & $12(54.5)$ \\
\hline Age at cART initiation (years), median (IQR) & $3(1.25-8.6)$ \\
\hline \multicolumn{2}{|l|}{ Age categories (years), $n$ (\%) } \\
\hline $0-3$ & $10 / 20(50.0)$ \\
\hline $3.1-5$ & $2 / 20(10.0)$ \\
\hline $5.1-10$ & $5 / 20(25.0)$ \\
\hline $10.1-16$ & $3 / 20(15.0)$ \\
\hline Unknown & $2(9.1)$ \\
\hline \multicolumn{2}{|l|}{ WHO stage, $n(\%)$} \\
\hline I & $1(4.5)$ \\
\hline II & $1(4.5)$ \\
\hline III & $17(77.3)$ \\
\hline IV & $3(13.6)$ \\
\hline \multicolumn{2}{|l|}{ TB treatment history, $n(\%)$} \\
\hline Yes & $14(63.6)$ \\
\hline No & $8(36.4)$ \\
\hline Nadir CD4+ T-cell count (cells/ $\mu \mathrm{L})$, median (IQR) & $289(94-687)$ \\
\hline \multicolumn{2}{|l|}{ CD4+ T-cell count categories (cells/ $\mu \mathrm{L}), n(\%)$} \\
\hline$<50$ & $4(21)$ \\
\hline $50-100$ & $2(10.5)$ \\
\hline $101-200$ & $1(5.3)$ \\
\hline $201-300$ & $3(15.8)$ \\
\hline $301-500$ & $1(5.3)$ \\
\hline $501-1000$ & $7(36.8)$ \\
\hline$>1000$ & $1(5.3)$ \\
\hline Unknown & $3(13.6)$ \\
\hline Nadir CD4+ T-cell percentage (cells/ $\mu \mathrm{L}$ ), median (IQR) & $10.3(1.97-17.5)$ \\
\hline \multicolumn{2}{|l|}{ CD4+ T-cell percentage, $n(\%)$} \\
\hline $0-10$ & $9(47.3)$ \\
\hline $11-20$ & $9(47.3)$ \\
\hline $20-30$ & $1(5.3)$ \\
\hline $30-50$ & 0 \\
\hline Unknown & $3(13.6)$ \\
\hline
\end{tabular}




\begin{tabular}{|c|c|}
\hline Characteristic & All children $(N=22)$ \\
\hline Age (years), median (IQR) & $8(4.35-11.7)$ \\
\hline \multicolumn{2}{|l|}{ Age categories (years), $n$ (\%) } \\
\hline $0-3$ & $1(5)$ \\
\hline $3.1-5$ & $6(30)$ \\
\hline $5.1-10$ & $7(35)$ \\
\hline $10.1-16$ & $6(30)$ \\
\hline Unknown & $2(9.1)$ \\
\hline CD4+ T-cell percentage, median (IQR) & $18.7(14-23.7)$ \\
\hline \multicolumn{2}{|l|}{ CD4+ T-cell percentage, $n(\%)$} \\
\hline $0-10$ & $3(16.7)$ \\
\hline $11-20$ & $7(38.9)$ \\
\hline $21-30$ & $6(33.3)$ \\
\hline$>30$ & $2(11.1)$ \\
\hline Unknown & $4(18.2)$ \\
\hline VL $\left(\log _{10}\right.$ copies/mL), median (IQR) & $4.52(3.38-4.8)$ \\
\hline Duration of cART (years), median (IQR) & $3(0.95-6.94)$ \\
\hline Duration of failing therapy (VF) (months), median (IQR) & $22(6-66)$ \\
\hline \multicolumn{2}{|l|}{ ART regimens, $n(\%)$} \\
\hline AZT, 3TC, LPV/r & $7(31.8)$ \\
\hline $\mathrm{d} 4 \mathrm{~T}, 3 \mathrm{TC}, \mathrm{LPV} / \mathrm{r}$ & $3(13.6)$ \\
\hline $\mathrm{AZT}, \mathrm{ABC}, \mathrm{LPV} / \mathrm{r}$ & $5(22.7)$ \\
\hline $\mathrm{ABC}, 3 \mathrm{TC}, \mathrm{LPV} / \mathrm{r}$ & $7(31.8)$ \\
\hline
\end{tabular}

and 6 (27.3\%) harbouring 1, 2 and $\geq 3$ TAMs, respectively. A distinct TAMs 1 pathway pattern was detected in 3 patients, with the rest displaying overlapping TAMs 1 and 2 pathways. Non-nucleoside reverse transcriptase inhibitor (NNRTI) mutations were detected in 14 children $(63.6 \%)$, at the following frequencies: $K 103 \mathrm{~N} n=5$, 22.7\%; V106M n=3, 13.6\%; G190A $n=5,22.7 \%$; Y188C/L $n=5,22.7 \%$; and $E 138 \mathrm{~A} / \mathrm{Q} n=2,9.1 \%$. Cross-level resistance to first-generation NNRTIs was evident in 12 of these children (85.7\%), with only 7 of them remaining fully susceptible to etravirine, a second-generation NNRTI often used for construction of salvage therapy.

\section{Discussion}

With high levels of virological failure in our cohort, convenience sampling of 236 children for HIV genotyping was conducted over a 6-year period. We focused on the $9.3 \%(22 / 236)$ who displayed PI resistance mutations and were on PIs at the time of HIV genotyping. Our findings of increasing PI resistance mutations are consistent with other SA studies of similar size conducted between 2000 and 2012 , in which rates of PI resistance mutations were $6.3 \%{ }^{[15]} 10.7 \%{ }^{[14]}$ and $14 \% .{ }^{[16]}$ However, another study reported a high prevalence of major PI mutations $(49 \%)^{[17]}$ in a public sector setting in SA.

Baseline clinical information on the study participants reflects a pattern consistent with poor outcomes. ${ }^{[18]}$ While the overall median age of initiation of cART was 3 years, $13.6 \%$ of the children were initiated at ages between 10 and 16 years and $91.0 \%$ were clinically staged as WHO III or IV. In addition, $40.9 \%$ of the children had a nadir CD4+ T-cell percentage between $0 \%$ and $10 \%$. It has been suggested and shown that a low CD4+ T-cell count may be associated with an increased probability of virological failure on PI-based regimens. ${ }^{[19-21]}$

A factor contributing to the emergence of major PI resistance mutation is prior exposure to an unboosted PI during ongoing viraemia. ${ }^{[17,22,23]}$ With the SA national antiretroviral treatment guidelines in the past (2004 - 2007) having advocated full-dose RTV in children aged $<6$ months and those on concomitant rifampicinbased antituberculosis therapy, ${ }^{[12]} 6(27.3 \%)$ of the children in our cohort with major PI mutations had been on full-dose RTV. Similar findings from a number of small studies of children on full-dose RTV support the development of major PI resistance mutations, with reported rates of $37.1 \%,{ }^{[24]} 50 \%{ }^{[18]}$ and $72 \%{ }^{[14]}$ However, the high proportion of children with major PI-associated resistance mutations in our cohort (95.5\%) cannot be explained by prior use of RTV alone. Three of the 21 children with major PI mutations (14.3\%) also had natural polymorphisms that on their own would not be expected to affect the efficacy of boosted PIs. ${ }^{[25-27]}$

Knowledge of the effect of various HIV resistance mutations on antiretroviral susceptibility can facilitate selection of the most active second- or third-line regimen, especially if options involving other classes of drugs have been exhausted. In our cohort, major PI mutations were detected at a high rate of $95.5 \%$, resulting in $66.7 \%$ of children losing activity of both boosted LPV and ATV, with 12 (57.1\%) remaining fully susceptible to second-generation DRV. V82A - a non-polymorphic mutation selected primarily by boosted LPV - was one of the most frequently occurring PI mutations in this cohort, detected in $76.2 \%$ of the children with major PI mutations. Emergence of V82A resulted in loss of activity of boosted LPV with cross-level resistance to boosted ATV. ${ }^{[28-30]}$ However, L76V, a PI mutation selected for by LPV and DRV, ${ }^{[31,32]}$ was observed in 7 (33.3\%) of these children, resulting in loss of activity of both boosted LPV and DRV. The presence of the major PI mutations with resulting loss to both LPV and ATV, as well as DRV, accessible only through the SA National Department of Health third-line committee as part of salvage therapy, remains a serious concern. The fact that none of the children in our cohort were presented to the third-line committee 
highlights the need for increased awareness of availability of access to this committee.

\section{Study limitations}

A limitation of this study is the fact that it was conducted at a single site, an academic hospital, so the findings may not be generalisable to other settings. The small cohort size also means that caution should be exercised when interpreting these findings, and a study with a much larger cohort may be needed to corroborate the findings.

\section{Conclusions}

Despite gains made in HIV management, HIV resistance continues to be a significant challenge in HIV-infected children, limiting current and future options. Of concern is the loss of antiretroviral agents typically used to construct a regimen for salvage therapy and the fact that none of the children in the study had accessed third-line committee-recommended therapy.

Declaration. The publication of this article was a requirement for ZM's $\mathrm{PhD}$ degree.

Acknowledgements. We acknowledge the children and staff at DGMAH. Author contributions. ZM contributed to the study design, analysis and writing of the manuscript. JTB, SM and OT contributed to the study design, analysis and manuscript review. PM and HM largely contributed to the manuscript review.

Funding. We thank the Discovery Foundation Fellowship Award for financial contribution towards this study.

Conflicts of interest. None.

1. Sutcliffe CG, van Dijk JH, Bolton C, Persaud D, Moss WJ. Effectiveness of antiretroviral therapy among HIV-infected children in sub-Saharan Africa. Lancet Infect Dis 2008;8(8):477-489. https://doi. among HIV-infected children in sub-S

2. World Health Organization, United Nations Children's Fund, Joint United Nations Programme on HIV and AIDS. Global Update on HIV Treatment 2013: Results, Impact and Opportunities. HIV Treatment. Geneva: WHO, 2013. https://www.who.int/hiv/pub/progressreports/update2013/en/ (accessed 25 August 2018)

3. Sohn AH, Hazra R. The changing epidemiology of the global paediatric HIV epidemic: Keeping rack of perinatally HIV-infected adolescents. J Int AIDS Soc 2013;16:18555. https://doi.org/10.7488/ IAS.16.1.18555

4. Meyers T, Moultrie H, Naidoo K, Cotton M, Eley B, Sherman G. Challenges to pediatric HIV care and treatment in South Africa. J Infect Dis 2007;196(Suppl 3):S474-S481. https://doi.org/10.1086/521116

5. Borghi J, Ataguba J, Mtei G, et al. Methodological challenges in evaluating health care financing equity in data-poor contexts: Lessons from Ghana, South Africa and Tanzania. In: Chernichovsky D, Hanson $\mathrm{K}$, eds. Innovations in Health System Finance in Developing and Transitional Economies (Advances in Health Economics and Health Services Research, vol. 21). Bingley, UK: Emerald Group Publishing, 2009:133-156.

6. Meyers TM, Yotebieng M, Kuhn L, Moultrie H. Antiretroviral therapy responses among children attending a large public clinic in Soweto, South Africa. Pediatr Infect Dis J 2011;30(11):974-979. attending a large public clinic in Soweto, South
https://doi.org/10.1097/INF.0b013e31822539f6

7. Sigaloff KC, Calis JC, Geelen SP, van Vugt M, de Wit TF. HIV-1-resistance-associated mutations after ailure of first-line antiretroviral treatment among children in resource-poor regions: A systematic review. Lancet Infect Dis 2011;11(10):769-779. https://doi.org/10.1016/S1473-3099(11)70141-4

8. Gupta RK, Gibb DM, Pillay D. Management of paediatric HIV-1 resistance. Curr Opin Infect Dis 2009;22(3):256-263. https://doi.org/10.1097/QCO.0b013e3283298f1f

9. Janssen N, Ndirangu J, Newell ML, Bland RM. Successful paediatric HIV treatment in rural primary care in Africa. Arch Dis Child 2010;95(6):414-421. https://doi.org/10.1136/adc.2009.16936
10. Green TN, Archary M, Gordon ML, et al. Drug resistance and coreceptor usage in HIV type 1 subtype $\mathrm{C}$-infected children initiating or failing highly active antiretroviral therapy in South Africa. AIDS Res C-infected children initiating or failing highly active antiretroviral therapy in

11. National Department of Health, South Africa. National Consolidated Guidelines for Prevention of National Department of Health, South Africa. National Consolidated Guidelines for Prevention of Mother-to-Child Transmission of HIV (PMTCT) and the Management of Children, Adolescents and 7 June 2017)

12. National Department of Health, South Africa. National Antiretroviral Treatment Guidelines. 2004. http://apps.who.int/medicinedocs/documents/s17758en/s17758en.pdf (accessed 26 May 2019).

13. Fatti G, Bock P, Grimwood A, Eley B. Increased vulnerability of rural children on antiretroviral therapy attending public health facilities in South Africa: A retrospective cohort study. J Int AIDS Soc 2010;13:46. https://doi.org/10.1186/1758Pe-2652-13-46

14. Orrell C, Levison J, Ciaranello A, et al. Resistance in pediatric patients experiencing virologic failure with first-line and second-line antiretroviral therapy. diatr Infect Dis J 2013;32(6):644-647. https://doi. org/10.1097/INF.0b013e3182829092

15. Pillay S, Bland RM, Lessells RJ, Manasa J, de Oliveira T, Danaviah S. Drug resistance in children a virological failure in a rural KwaZulu-Natal, South Africa, cohort. AIDS Res Ther 2014;11(1):3. https:/ doi.org/10.1186/1742-6405-11-3

16. Rossouw TM, Feucht UD, Melikian G, et al. Factors associated with the development of drug resistance mutations in HIV-1 infected children failing protease inhibitor-based antiretroviral therapy in South Africa. PLoS One 2015;10(7):e0133452. http://doi.org/10.1371/journal.pone.0133452

17. Le Moing V, Chene G, Carrieri MP, et al. Predictors of virological rebound in HIV-1-infected patients initiating a protease inhibitor-containing regimen. AIDS 2002;16(1):21-29. https://doi. org/10.1097/00002030-200201040-00004

18. Nuttall J, Pillay V. Characteristics and early outcomes of children and adolescents treated with darunavir/ritonavir-, raltegravir- or etravirine-containing antiretroviral therapy in the Western Cape Province of South Africa. S Afr Med J 2018;108(2):105-110. https://doi.org/10.7196/SAMJ2018. v108i2.12573

19. Ananworanich J, Kosalaraksa P, Siangphoe U, et al. A feasibility study of immediate versus deferrec antiretroviral therapy in children with HIV infection. AIDS Res Ther 2008;5:24. https://doi. org/10.1186/1742-6405-5-24

20. Paredes R, Mocroft A, Kirk O, et al. Predictors of virological success and ensuing failure in HIVpositive patients starting highly active antiretroviral therapy in Europe: Results from the EuroSIDA positive patients starting highly active antiretroviral therapy in Europe: Results from the
study. Arch Intern Med 2000;160(8):1123-1132. https://doi.org/10.1001/archinte. 160.8 .1123

21. Grabar S, Pradier C, le Corfec E, et al. Factors associated with clinical and virological failure in Grabar S, Pradier C, le Corfec E, et al. Factors associated with clinical and virological failure in
patients receiving a triple therapy including a protease inhibitor. AIDS 2000;14(2):141-149. https://doi. org/10.1097/00002030-200001280-00009

22. Van Zyl GU, van der Merwe L, Claassen M, et al. Protease inhibitor resistance in South African children with virologic failure. Pediatr Infect Dis J 2009;28(12):1125-1127. https://doi.org/10.1097/ INF.0b013e3181af829

23. Van Zyl GU, van Mens TE, McIlleron H, et al. Low lopinavir plasma or hair concentrations explain second-line protease inhibitor failures in a resource-limited setting. J Acquir Immune Defic Synd 2011;56(4):333-339. https://doi.org/10.1097/QAI.0b013e31820dc0c

24. Taylor BS, Hunt G, Abrams EJ, et al. Rapid development of antiretroviral drug resistance mutations in HIV-infected children less than two years of age initiating protease inhibitor-based therapy in South Africa. AIDS Res Hum Retroviruses 2011;27(9):945-956. https://doi.org/10.1089/aid.2010.0205

25. Brumme CJ, Harrigan PR. No inherent association between minor mutations in HIV protease at baseline and selection of the $L 90 M$ mutation at the time of the first virological failure. J Infect Dis 2005;191(10):1778-1779; author reply 9-80. https://doi.org/10.1086/429672

26. Champenois K, Deuffic-Burban S, Cotte L, et al. Natural polymorphisms in HIV-1 protease: Impact on effectiveness of a first-line lopinavir-containing antiretroviral therapy regimen. J Med Viro 2008;80(11):1871-1879. https://doi.org/10.1002/jmv.21315

27. Frater AJ, Beardall A, Ariyoshi K, et al. Impact of baseline polymorphisms in RT and protease on outcome of highly active antiretroviral therapy in HIV-1-infected African patients. AIDS 2001;15(12):1493-1502. https://doi.org/10.1097/00002030-200108170-00006

28. Rhee SY, Liu T, Ravela J, Gonzales MJ, Shafer RW. Distribution of human immunodeficiency virus type 1 protease and reverse transcriptase mutation patterns in 4,183 persons undergoing genotypic resistance testing. Antimicrob Agents Chemother 2004;48(8):3122-3126. https://doi.org/10.1128/ AAC.48.8.3122-3126

29. Vermeiren H, van Craenenbroeck E, Alen P, et al. Prediction of HIV-1 drug susceptibility phenotype from the viral genotype using linear regression modeling. J Virol Methods 2007;145(1):47-55. https:// doi.org/10.1016/.j.jiromet.2007.05.009

30. Kempf DJ, Isaacson JD, King MS, et al. Identification of genotypic changes in human immunodeficiency virus protease that correlate with reduced susceptibility to the protease inhibitor lopinavir among vira virus protease that correlate with reduced susceptibility to the protease inhibitor lopinavir among viral
isolates from protease inhibitor-experienced patients. J Virol 2001;75(16):7462-7469. https://doi. org/10.1128/JVI.75.16.7462-7469.2001

31. Barber TJ, Harrison L, Asboe D, et al. Frequency and patterns of protease gene resistance mutation Barber TJ, Harrison L, Asboe D, et al. Frequency and patterns of protease gene resistance mutations
in HIV-infected patients treated with lopinavir/ritonavir as their first protease inhibitor. J Antimicrob in HIV-infected patients treated with lopinavir/ritonavir as their first
Chemother 2012;67(4):995-1000. https://doi.org/10.1093/jac/dkr569

32. Nijhuis M, Wensing AM, Bierman WF, et al. Failure of treatment with first-line lopinavir boosted with ritonavir can be explained by novel resistance pathways with protease mutation $76 \mathrm{~V}$. J Infect Dis 2009;200(5):698-709. https://doi.org/10.1086/605329 